\title{
TMDlib2 and TMDplotter: a platform for 3D hadron structure studies
}

\author{
N. A. Abdulov ${ }^{1}$, A. Bacchetta ${ }^{2}$, S. Baranov ${ }^{3}$, A. Bermudez Martinez ${ }^{4, a}$, V. Bertone ${ }^{5}$, C. Bissolotti ${ }^{2,6}$, V. Candelise ${ }^{7,8}$, \\ L. I. Estevez Banos ${ }^{4}$, M. Bury ${ }^{9}$, P. L. S. Connor ${ }^{4,21}$, L. Favart $^{10}$, F. Guzman ${ }^{11}$, F. Hautmann ${ }^{12,13}$, M. Hentschinski ${ }^{14}$, \\ H. Jung ${ }^{4, b}$, L. Keersmaekers ${ }^{12}$, A. Kotikov ${ }^{15}$, A. Kusina ${ }^{16}$, K. Kutak ${ }^{16}$, A. Lelek ${ }^{12}$, J. Lidrych ${ }^{4}$, A. Lipatov ${ }^{1}$, \\ G. Lykasov ${ }^{15}$, M. Malyshev ${ }^{1}$, M. Mendizabal ${ }^{4}$, S. Prestel ${ }^{17}$, S. Sadeghi Barzani ${ }^{12,18}$, S. Sapeta $^{16}$, M. Schmitz ${ }^{4}$, \\ A. Signori ${ }^{2,19}$, G. Sorrentino ${ }^{7,8}$, S. Taheri Monfared ${ }^{4}$, A. van Hameren ${ }^{16}$, A. M. van Kampen ${ }^{12}$,

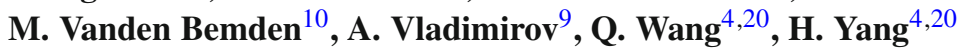 \\ ${ }^{1}$ SINP, Moscow State University, Moscow, Russia \\ 2 Dipartimento di Fisica, Università di Pavia and INFN, Pavia, Italy \\ ${ }^{3}$ Lebedev Physics Institute, Moscow, Russia \\ ${ }^{4}$ DESY, Hamburg, Germany \\ ${ }^{5}$ IRFU, CEA, Université Paris-Saclay, Gif-sur-Yvette, France \\ ${ }^{6}$ EIC Center, Jefferson Lab, Newport News, USA \\ ${ }^{7}$ INFN Sezione di Trieste, Trieste, Italy \\ ${ }^{8}$ Università di Trieste, Trieste, Italy \\ ${ }^{9}$ Institut für Theoretische Physik, Universität Regensburg, Regensburg, Germany \\ ${ }^{10}$ Inter-University Institute For High Energies, Universite Libre de Bruxelles, Brussels, Belgium \\ ${ }^{11}$ InSTEC, Universidad de La Habana, Havana, Cuba \\ 12 Elementary Particle Physics, University of Antwerp, Antwerp, Belgium \\ ${ }^{13}$ RAL and University of Oxford, Oxford, UK \\ ${ }^{14}$ Departamento de Actuaria, Fisica y Matemáticas, Universidad de las Américas, Puebla, Mexico \\ 15 JINR, Dubna, Russia \\ ${ }^{16}$ Institute of Nuclear Physics, Polish Academy of Sciences, Cracow, Poland \\ 17 Department of Astronomy and Theoretical Physics, Lund University, Lund, Sweden \\ 18 Department of Physics, Shahid Beheshti University, Tehran, Iran \\ 19 Theory Center, Jefferson Lab, Newport News, USA \\ ${ }^{20}$ School of Physics, Peking University, Beijing, China \\ ${ }^{21}$ Present address: University of Hamburg, Hamburg, Germany
}

Received: 25 March 2021 / Accepted: 29 July 2021 / Published online: 20 August 2021

(C) The Author(s) 2021

\begin{abstract}
A common library, TMDlib2, for TransverseMomentum-Dependent distributions (TMDs) and unintegrated parton distributions (uPDFs) is described, which allows for easy access of commonly used TMDs and uPDFs, providing a three-dimensional (3D) picture of the partonic structure of hadrons. The tool TMDplotter allows for webbased plotting of distributions implemented in TMDlib2, together with collinear pdfs as available in LHAPDF.
\end{abstract}

\section{Program summary}

Computer for which the program is designed and others on which it is operable: any with standard $\mathrm{C}++$, tested on Linux and Mac OS systems

\footnotetext{
a e-mail: armando.bermudez.martinez@desy.de

b e-mail: hannes.jung@desy.de (corresponding author)
}

Programming Language used: $\mathrm{C}++$ High-speed storage required: No

Separate documentation available: No

Other programs used: LHAPDF (version 6) for access to collinear parton distributions, ROOT (any version $>5.30$ ) for plotting the results

Download of the program: http://tmdlib.hepforge.org Unusual features of the program: None

Citation policy: please cite the current version of the manual and the paper(s) related to the parameterization(s).

\section{Introduction}

The calculation of processes at high energy hadron colliders is based in general on the calculation of a partonic process 
(matrix element) convoluted with the likelihood to find a parton of specific flavor and momentum fraction at a given scale within the hadrons. If the parton density depends only on the longitudinal momentum fraction $x$ of the hadron's momentum carried by a parton, and the resolution scale $\mu$, the processes are described by collinear factorization with the appropriate evolution of the parton densities (PDFs) given by the Dokshitzer-Gribov-Lipatov-Altarelli-Parisi (DGLAP) evolution equations [1-5]. Such descriptions are successful for sufficiently inclusive processes, like inclusive deep-inelastic lepton-hadron scattering (DIS).

In several less inclusive processes, also the transverse momentum of the interacting partons plays an important role, leading to an extension of the collinear factorization theorem to include transverse degrees of freedom. Different factorization theorems for the inclusion of transverse momenta to the parton densities have been developed in the past, leading to so-called Transverse Momentum Dependent (TMD) parton densities and unintegrated parton densities (uPDFs) [6]. These densities provide a 3D imaging of hadron structure, extending the 1D picture given by PDFs. For semi-inclusive processes, like semi-inclusive DIS (SIDIS), Drell-Yan (DY) production and $e^{+} e^{-}$scattering, TMD factorization has been formulated [7-19]. The high-energy (small- $x$ limit) factorization was formulated for heavy flavor and heavy boson production in Refs. [20-26] using unintegrated gluon distributions [27-35]. In Refs. [36,37] the Parton Branching (PB) method was formulated as a way to obtain TMD distributions for all flavours over a wide range of $x$, transverse momentum $k_{t}$, and scale $\mu$ essentially by solving next-to-leadingorder (NLO) DGLAP equations through Sudakov form factors, separating resolvable and non-resolvable branchings via the notion of soft-gluon resolution scale [38,39], and keeping track of the transverse momenta at each branching.

Since the number of available TMD densities increases very rapidly, and different groups provide different sets, it was necessary to develop a common platform to access the different TMD sets in a common form. In 2014 the first version of TMDlib (version 1) and TMDplotter was released [40,41], which made several TMD sets available to the community. This library has set a common standard for accessing TMD sets, similar to what was available for collinear parton densities in PDFlib [42,43] and LHAPDF [44]. TMDlib is a $\mathrm{C}++$ library which provides a framework and an interface to a collection of different UPDF and TMD parameterizations.

In this report, we describe a new version of the TMDlib library, TMDlib2, as well as the associated online plotting tool TMDplotter. TMDlib2 covers all the features present already in the previous version and contains significant new developments, such as the treatment of TMD uncertainties and a more efficient method to include new TMD sets. The report is structured as follows. In Sect. 2, we give the main elements of the library framework. In Sect. 3 we emphasize the new features of TMDlib2 compared to the previous version. In Sect. 4 we provide the essential documentation. We summarize in Sect. 5.

\section{The TMDlib framework}

The TMDlib library and its new version TMDlib2 consider momentum weighted TMD parton distributions $x \mathcal{A}_{j}\left(x, k_{t}, \mu\right)$ of flavor $j$ as functions of the parton's light cone longitudinal momentum fractions $x$ of the hadron's momentum, the parton's transverse momentum $k_{t}$, and the evolution scale $\mu$ [6]. Besides, the library also contains integrated TMDs obtained from the integration over $k_{t}$, as follows

$x \mathcal{A}_{\text {int }}(x, \mu)=\int_{k_{t, \text { min }}}^{k_{t, \max }} d k_{t}^{2} x \mathcal{A}\left(x, k_{t}, \mu\right)$,

In Fig. 1 (left), we show an example of integrated TMD obtained with TMDplotter for the PB-NLO-HERAI+II2018-set1 [45], in which the integral between $k_{t, \min }=0.01$ and $k_{t, \text { max }}=100 \mathrm{GeV}$ is compared with the collinear PDF set HERAPDF2.0 [46]. By construction both sets are identical. However, in general, Eq. (1) does not converge to the collinear pdf, which is shown in Fig. 1 (right) comparing the integral between $k_{t, \min }=0.01$ and $k_{t, \max }=100$ GeV of PV17 [47] with the corresponding collinear distribution of MMHT2014 [48]. Several aspects of the relationship between integrated TMDs and collinear PDFs have been investigated in the literature. The matching coefficient between the integrated gluon TMD and the collinear gluon PDF in the MSbar scheme was first computed in the small- $x$ limit in Ref. [24], with small- $x$ resummation of logarithmic accuracy $\left(\alpha_{s} \ln x\right)^{m}$ to all orders $m$ in $\alpha_{s}$. Perturbative calculations of the matching coefficients at finite order have recently been carried out through $\mathrm{N}^{3} \mathrm{LO}$ in Refs. [49,50]. Other aspects of the relationship between integrated TMDs and collinear PDFs are studied e.g. in [21,27,28,51-54]. We refer the reader to the overview [6], and references therein, for further discussions of this topic.

In TMDlib2 the densities are defined more generally as momentum weighted distributions $x \mathcal{A}\left(x, \bar{x}, k_{t}, \mu\right)$, where $x, \bar{x}$ are the (positive and negative) light-cone longitudinal momentum fractions [51,54-56]. In some of the applications $\bar{x}$ is set explicitly to zero, while in other cases $\bar{x}=0$ means that it is implicitly integrated over.

\subsection{Grids and Interpolation}

Since the analytic calculation of TMDs as a function of the longitudinal momentum fraction $x$ (we neglect $\bar{x}$ in the following), the transverse momentum $k_{t}$ and the scale $\mu$ is very time consuming and in some cases even not available, the TMDs are saved as grids, and TMDlib provides appropri- 


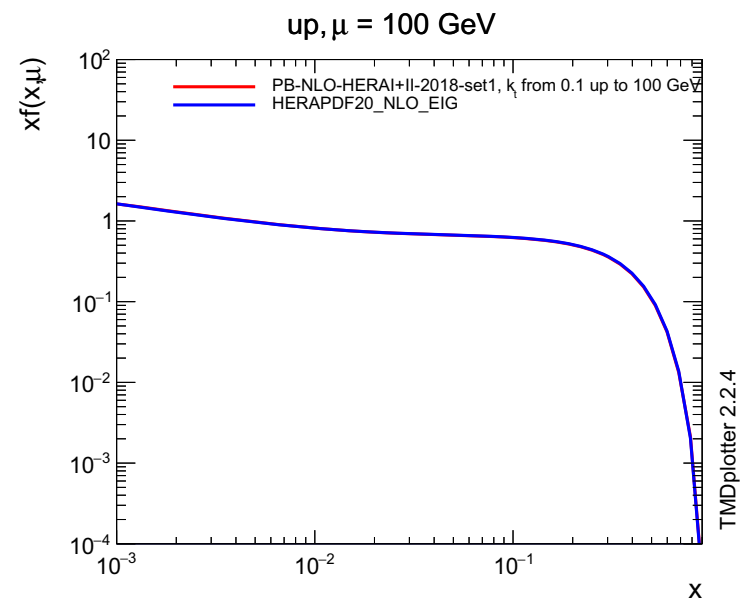

Fig. 1 Comparison of up-type parton distributions, $x f(x, \mu)=$ $x \mathcal{A}_{\text {int }}(x, \mu)$ as a function of $x$ at $\mu=100 \mathrm{GeV}$ (left): comparison of the integrated distribution PB-NLO-HERAI+II-2018-set1 [45] with HER-

ate tools for interpolation between the grid points (where the type of evolution is indicated):

\begin{tabular}{lc}
\hline allFlavPDF & Multidimensional Linear Interpolation in $x$, \\
& $k_{t}$ and $\mu$ is used for PB and CCFM-type \\
& TMDs \\
Pavia & Interpolation based on Lagrange \\
& polynomials of degree three, performed \\
& through APFEL++ [57,58] \\
InterpolationKS & Multidimensional cubic spline interpolation \\
& in $x, k_{t}$ and $\mu$, based on GSL \\
& implementation, is used for KS-type \\
& TMDs (see Table 1) \\
\hline
\end{tabular}

The parameterizations of TMDs in TMDlib are explicitly authorized for each distribution by the corresponding authors. A list of presently available TMD sets is given in Table 1. No explicit QCD evolution code is included: the parameterizations are as given in the corresponding references.

The grids of each selected TMD set are read into memory once (the I/O time depends on the size of the grid). Each TMD set is initialized as a separate instance of the TMD class, which is created for each different TMD set, for example for uncertainty sets, or if several different TMD sets are needed for the calculation. The memory consumption of TMDlib is determined by the size of the TMDgrids. Optionally, TMDgrids can be loaded separately, avoiding large memory consumption.

It is the philosophy of TMDlib that the definition of TMD grids is left free, but a few examples are given: the grids for the PB, CCFM and KS TMD sets are stored in form of text tables, the grids of the Pavia type TMDs are stored and read via the YAML frame. The method of interpolation and

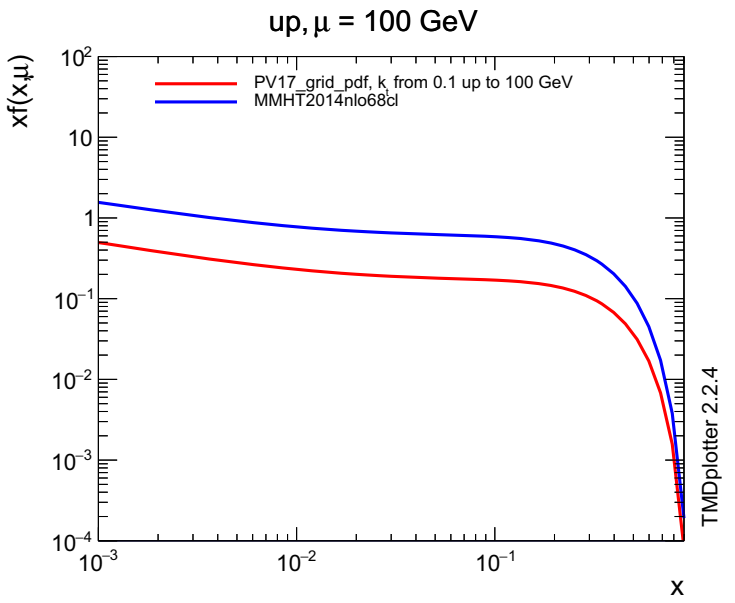

APDF2.0 [46]. (right): comparison of integrated distribution PV17 [47] with MMHT2014 [48]

the corresponding accuracy of the interpolation is left under control of the authors of the relevant TMD sets.

\subsection{Uncertainty TMD sets}

The estimation of theoretical uncertainties is an important ingredient for phenomenological applications, and uncertainties from PDFs and TMDs play a central role. The uncertainties of TMDs are estimated usually from the uncertainties of the input parameters or parameterization. There are two different methods commonly used: the Hessian method [59] which is applied if the parameter variations are orthogonal or the Monte Carlo method providing Monte Carlo replicas $[60,61]$. The specific prescriptions on how to calculate the uncertainties for a given TMD set should be found in the original publication describing the TMDs.

An example of TMDs with uncertainty band is shown in Fig. 2 for the PB set as well as for the PV17 set. The parameters of intrinsic $k_{t}$ - distribution are part of the fit of PV17, while they are not fitted for the PB sets (see discussion in Ref. [45]).

\subsection{TMDplotter}

TMDlib provides also a web-based application for plotting TMD distributions - TMDplotter, plotting tools for collinear pdfs are available under e.g. [62] or [63]. In Fig. 3 (left) a comparison of the transverse momentum distributions of different TMD sets is shown, and in Fig. 3 (right) the gluongluon luminosity calculation for the integrated TMD sets PB-NLO-HERAI+II-2018-set1 [45] at $\mu=100 \mathrm{GeV}$ compared with the one obtained from HERAPDF 2.0 is shown (the curves obtained from PB-NLO-HERAI+II-2018-set1 and HERAPDF 2.0 overlap). 
Table 1 Available uPDF/TMD parton sets in TMDlib

\begin{tabular}{|c|c|c|c|}
\hline iset & uPDF/TMD set & Subsets & References \\
\hline 101000 & CCfm-JS-2001 & 1 & {$[65]$} \\
\hline 101010 & $\operatorname{ccfm}-\operatorname{set} \mathrm{A} 0$ & 4 & {$[65]$} \\
\hline 101020 & ccfm-setB0 & 4 & {$[65]$} \\
\hline 101001 & $\mathrm{CCfm}-\mathrm{JH}-\mathrm{set} 1$ & 1 & {$[66]$} \\
\hline 101002 & $\mathrm{cCfm}-\mathrm{JH}-\mathrm{set} 2$ & 1 & {$[66]$} \\
\hline 101003 & $\mathrm{ccfm}-\mathrm{JH}-\mathrm{set} 3$ & 1 & {$[66]$} \\
\hline 101201 & $\mathrm{ccfm}-\mathrm{JH}-2013-$ set 1 & 13 & {$[67]$} \\
\hline 101301 & $\mathrm{ccfm}-\mathrm{JH}-2013-$ set 2 & 13 & {$[67]$} \\
\hline 101401 & MD-2018 & 1 & {$[68]$} \\
\hline 101410 & KLSZ-2020 & 1 & [69] \\
\hline 102100 & PB-NLO-HERAI+II-2018-set 1 & 35 & {$[45]$} \\
\hline 102200 & $\mathrm{~PB}-\mathrm{NLO}-\mathrm{HERAI}+\mathrm{II}-2018-$ set 2 & 37 & [45] \\
\hline 102139 & $\mathrm{~PB}-\mathrm{NLO}-\mathrm{HERAI}+\mathrm{II}-2018-$ set $1-\mathrm{q} 0$ & 3 & {$[45]$} \\
\hline 102239 & $\mathrm{~PB}-\mathrm{NLO}-\mathrm{HERAI}+\mathrm{II}-2018-$ set $2-\mathrm{q} 0$ & 3 & {$[45]$} \\
\hline 103100 & $\mathrm{~PB}-\mathrm{NLO}+\mathrm{QED}-$ set $1-\mathrm{HERAI}+\mathrm{II}$ & 1 & {$[70]$} \\
\hline 103200 & $\mathrm{~PB}-\mathrm{NLO}+\mathrm{QED}-$ set $2-\mathrm{HERAI}+\mathrm{II}$ & 1 & {$[70]$} \\
\hline 10904300 & PB-NLO_ptoPb208-set1 & 1 & [71] \\
\hline 10904400 & PB-NLO_ptoPb208-set 2 & 1 & [71] \\
\hline 10901300 & PB-EPPS16n10_CT14n10_Pb208-set1 & 1 & {$[71]$} \\
\hline 10901400 & PB-EPPS16n10_CT14n1o_Pb208-set2 & 1 & [71] \\
\hline 10902300 & PB-nCTEQ15FullNuc_208_82-set1 & 33 & [71] \\
\hline 10902400 & PB-nCTEQ15Ful1Nuc_208_82-set2 & 33 & [71] \\
\hline 200001 & GBWlight & 1 & {$[72]$} \\
\hline 200002 & GBWcharm & 1 & {$[72]$} \\
\hline 210001 & Blueml & 1 & [73] \\
\hline 400001 & KS-2013-linear & 1 & {$[74]$} \\
\hline 400002 & KS-2013-non-linear & 1 & {$[74]$} \\
\hline 400003 & KS-hardscale-linear & 1 & {$[75]$} \\
\hline 400004 & KS-hardscale-non-linear & 1 & {$[75]$} \\
\hline 400101 & KS-WeizWill-2017 & 1 & {$[76]$} \\
\hline 500001 & EKMP & 1 & [77] \\
\hline 410001 & BHKS & 1 & [78] \\
\hline 300001 & SBRS-2013-TMDPDFs & 1 & [79] \\
\hline 300002 & SBRS-2013-TMDPDFs-par & 1 & [79] \\
\hline 601000 & PV17_grid_pdf & 201 & [47] \\
\hline 602000 & PV17_grid_ff_Pim & 201 & [47] \\
\hline 603000 & PV17_grid_ff_Pip & 201 & [47] \\
\hline 604000 & PV17_grid_FUUT_Pim & 100 & [47] \\
\hline 605000 & PV17_grid_FUUT_Pip & 100 & [47] \\
\hline 606000 & PV19_grid_pdf & 216 & {$[80]$} \\
\hline 607000 & PV20_grid_FUTTsin_P_Pim & 101 & {$[81]$} \\
\hline 608000 & PV20_grid_FUTTsin_P_Pip & 101 & [81] \\
\hline 701000 & SV19_nnlo & 23 & {$[82]$} \\
\hline 702000 & SV19_nnlo_all=0 & 21 & {$[82]$} \\
\hline 703000 & SV19_n310 & 23 & {$[82]$} \\
\hline
\end{tabular}


Table 1 continued

\begin{tabular}{llll}
\hline iset & uPDF/TMD set & Subsets & Ref. \\
\hline 704000 & SV19_n310_all=0 & 21 & [82] \\
705000 & SV19_ff_pi_n310 & 23 & [82] \\
706000 & SV19_ff_pi_n310_all=0 & 21 & [82] \\
707000 & SV19_ff_K_n3lo & 23 & {$[82]$} \\
708000 & SV19_ff_K_n310_all=0 & 21 & [82] \\
709000 & SV19_pion & 7 & [83] \\
710000 & SV19_pion_all=0 & 7 & [83] \\
711000 & BPV20_Sivers & 25 & {$[84]$} \\
\hline
\end{tabular}

up, PB-NLO-HERAI+II-2018-set2, $x=0.08, \mu=100 \mathrm{GeV}$

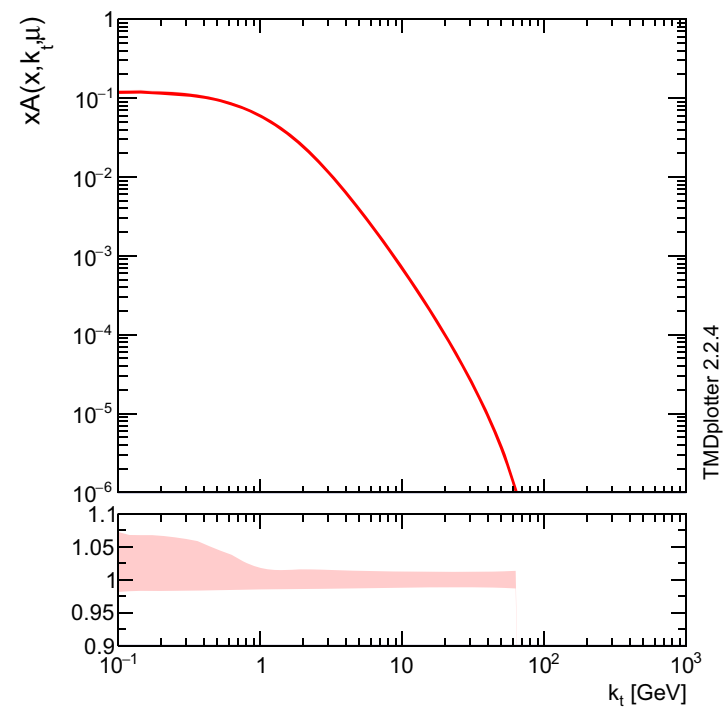

up, PV17_grid_pdf, $x=0.08, \mu=100 \mathrm{GeV}$

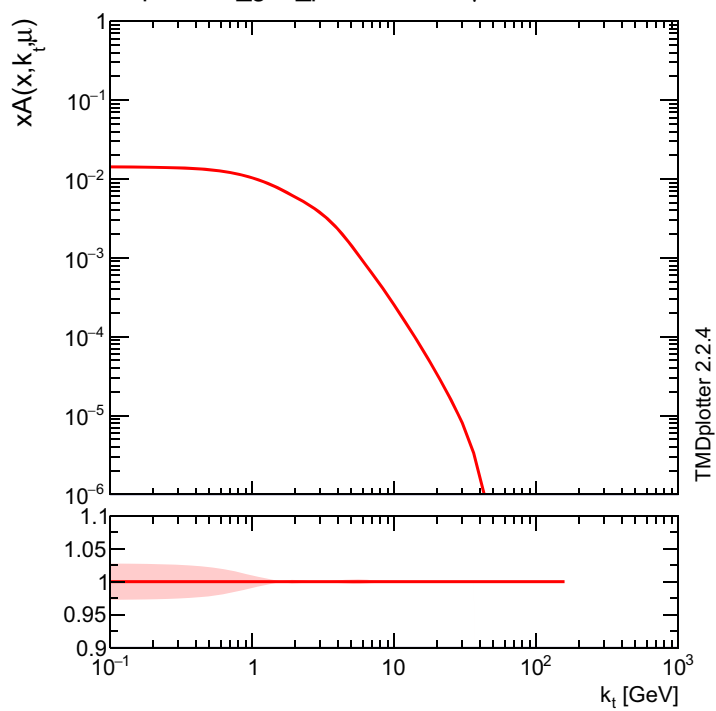

Fig. 2 Transverse momentum distribution $x \mathcal{A}\left(x, k_{t}, \mu\right)$ at $x=0.08$ and $\mu=100 \mathrm{GeV}$ obtained with PB-NLO-HERAI+II-2018-set2 [45] (left) and PV17 [47] (right)

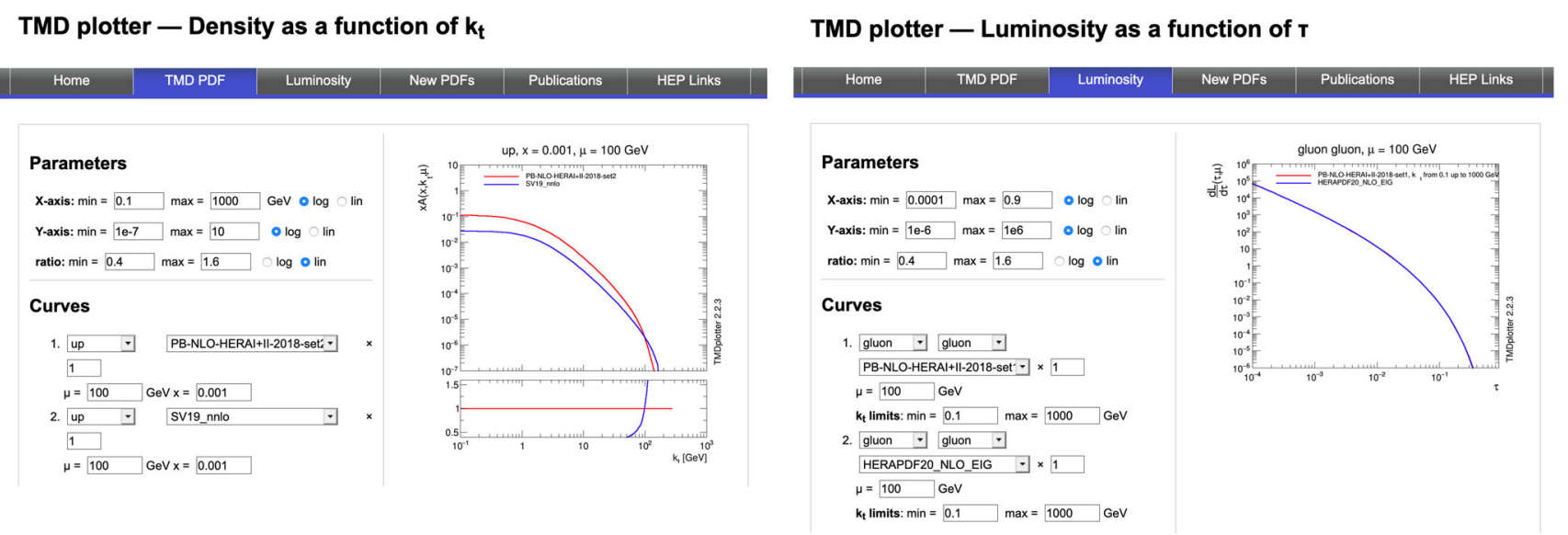

Fig. 3 TMDplotter examples: (left) comparison of the transverse momentum distributions of different TMD sets, (right) gluon-gluon luminosity calculation using integrated TMD sets (the curves from PB-NLO-HERAI+II-2018-set1 and HERAPDF2 . 0 overlap) 
TMDplotter is available at http://tmdplotter.desy.de/.

\section{New features}

Having described in Sect. 2 the general framework of the TMDlib library, we here stress the main new features of TMDlib2 compared with the previous version [40] of the library. The most important development concerns the inclusion of many new TMD sets. This is achieved through a new and more efficient method to add input files. The method is flexible enough that it will allow new sets, which may become available in the future, to also be included in a straightforward manner. Another extremely important development of TMDlib2, which plays an essential role in paving the way to systematic TMD phenomenology at colliders and fixed target machines, is that the uncertainties associated with TMD sets are now accessible through the library. This was not the case in the first version [40]. It is the first time that TMD uncertainties become available in a library tool. While uncertainties on collinear PDFs are nowadays available through several different web-based resources, TMDlib2 is at present the unique tool which contains the full existing information on uncertainties on TMD sets, and makes it readily accessible. As such, we expect it to be an essential tool for phenomenological studies of TMDs and comparisons with experimental data.

To sum up, the main new features of TMDlib2 compared to the earlier version of the library are as follows.

- TMDlib2 makes use of C++ classes, and the different sets corresponding to uncertainty sets or sets corresponding to different parameterizations are read once and initialized as different instances, allowing to load many sets into memory;

- information about TMD sets is read via YAML from the TMD info files, containing all metadata;

- including new TMD sets is simplified with the new structure of the input sets;

- the TMD sets are no longer part of the TMDlib distribution, but can be downloaded via TMDlib-getdata, distributed with TMDlib2.

\section{TMDlib documentation}

TMDlib is written in $\mathrm{C}++$, with an interface for access from FORTRAN code. The source code of TMDlib is available from http://tmdlib.hepforge.org/ and can be installed using the standard autotools sequence configure, make, make install, with options to specify the installation path and the location of the LHAPDF PDF library [44], and the ROOT data analysis framework library [64] (which is used optionally for plotting). If ROOT is not found via root-config, the plotting option is disabled. After installation, TMDlib-config gives access to necessary environment variables.

\subsection{Description of the program components}

Initialization in $\mathrm{C}++$

\begin{tabular}{ll}
\hline TMDinit (name) & $\begin{array}{l}\text { To initialize the dataset specified by } \\
\text { its name name. A complete list of } \\
\text { datasets available in the current ver- } \\
\text { sion of TMDlib with the correspond- } \\
\text { ing name is provided in Table 1 } \\
\text { To initialize a given irep } \\
\text { replica of the dataset name } \\
\text { To initialize the dataset speci- } \\
\text { fied by its identifier iset }\end{array}$ \\
\hline
\end{tabular}

Initialization in Fortran

TMDinit (iset)

To initialize the dataset specified by its identifier iset

TMDset (iset) 
Access to TMDs in $C++$
TMDpdf (x,
xbar, kt, $\mathrm{mu}$ )
TMDpdf (x, xbar, kt, mu, uval, dval, sea, charm, bottom, gluon, photon)

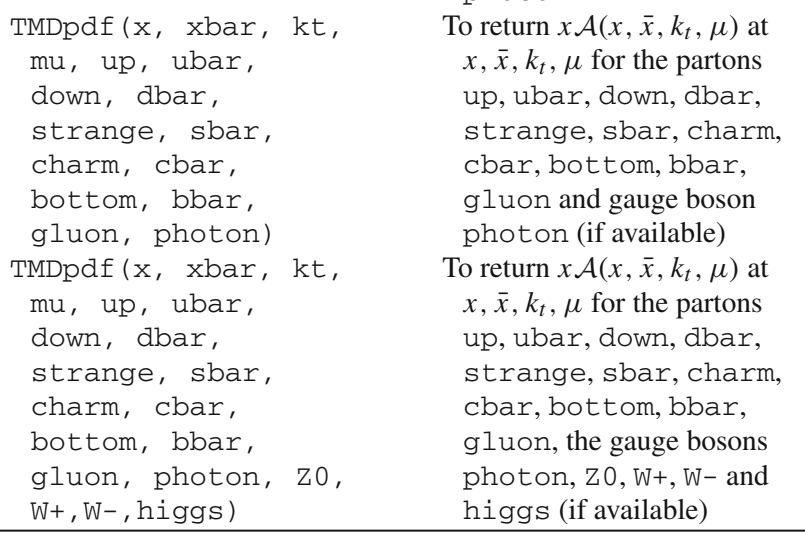

To return $x \mathcal{A}\left(x, \bar{x}, k_{t}, \mu\right)$ at $x, \bar{x}, k_{t}, \mu$ for the partons cbar, bottom, bbar, gluon and gauge boson ton (if available) up, ubar, down dbar, strange, sbar, charm, gluon, the gauge bosons higgs (if available)

Access to TMDs in Fortran
Vector double-type TMDpdf (kf, x, xbar,

function returning an

array of 13 variables

for QCD parton

densities with the

values of

$x \mathcal{A}\left(x, \bar{x}, k_{t}, \mu\right):$ at

index $0, \ldots, 5$ is

$\bar{t}, \ldots, \bar{d}$, at index 6

is the gluon, and at

index $7, \ldots, 12$ is

$d, \ldots, t$ densities

Void-type function filling an array of 13 variables, xpq, with the values of $x \mathcal{A}\left(x, \bar{x}, k_{t}, \mu\right)$ : at index $0, \ldots, 5$ is $\bar{t}, \ldots, \bar{d}$, at index 6 is the gluon, and at index $7, \ldots, 12$ is $d, \ldots, t$ densities

Void-type function to return $x \mathcal{A}\left(x, \bar{x}, k_{t}, \mu\right)$ at $x, \bar{x}, k_{t}, \mu$ for valence u-quarks uval, valence d-quarks dval, light sea-quarks $\mathrm{s}$, charm-quarks c, bottom-quarks b, gluons glu and gauge boson photon

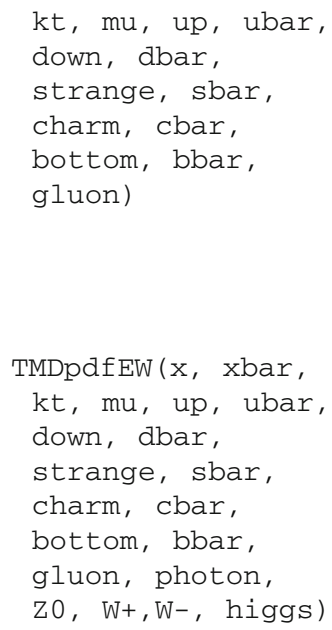

To return $x \mathcal{A}\left(x, \bar{x}, k_{t}, \mu\right)$ at $x, \bar{x}, k_{t}, \mu$ for the partons up, ubar, down, dbar, strange, sbar, charm, cbar, bottom, bbar, gluon for the hadron flavor $\mathrm{kf}$. ( $\mathrm{kf}$ is no longer used, only kept for backward compatibility with TMDlib1)

To return $x \mathcal{A}\left(x, \bar{x}, k_{t}, \mu\right)$ at $x, \bar{x}, k_{t}, \mu$ for the partons up, ubar, down, dbar, strange, sbar, charm, cbar, bottom, bbar, gluon, the gauge bosons photon, Z0, $\mathrm{W}+$, $\mathrm{W}$ - and higgs (if available)

\section{Callable program components}

The program components listed in this section are accessible with the same name in $\mathrm{C}++$ as well as in Fortran.

\begin{tabular}{|c|c|}
\hline TMDinfo (dataset) & $\begin{array}{l}\text { Accesses information from } \\
\text { the info file }\end{array}$ \\
\hline TMDgetDesc ( ) & $\begin{array}{l}\text { Returns data set description } \\
\text { from info file }\end{array}$ \\
\hline TMDget Index () & $\begin{array}{l}\text { Returns index number as a } \\
\text { string of data set from } \\
\text { info file }\end{array}$ \\
\hline TMDgetNumMembers ( ) & $\begin{array}{l}\text { Returns number of } \\
\text { members of data sets from } \\
\text { info file }\end{array}$ \\
\hline TMDgetScheme () & $\begin{array}{l}\text { Returns evolution scheme } \\
\text { of dataset from info file }\end{array}$ \\
\hline TMDgetNf ( ) & $\begin{array}{l}\text { Returns the number of } \\
\text { flavours, } N_{f} \text {, used for the } \\
\text { computation of } \Lambda_{Q C D}\end{array}$ \\
\hline TMDgetOrderAlphas ( ) & $\begin{array}{l}\text { Returns the perturbative } \\
\text { order of } \alpha_{\mathrm{s}} \text { used in the } \\
\text { evolution of the dataset }\end{array}$ \\
\hline TMDgetorderPDF ( ) & $\begin{array}{l}\text { Returns the perturbative } \\
\text { order of the evolution of } \\
\text { the dataset }\end{array}$ \\
\hline TMDgetXmin ( ) & $\begin{array}{l}\text { Returns the minimum value } \\
\text { of the momentum fraction } \\
x \text { for which the dataset } \\
\text { initialized by } \\
\text { TMDinit (name) was } \\
\text { determined }\end{array}$ \\
\hline
\end{tabular}




\begin{tabular}{|c|c|}
\hline TMDgetXmax ( ) & $\begin{array}{l}\text { Returns the maximum value } \\
\text { of the momentum fraction } \\
x \text { for which the dataset } \\
\text { initialized by } \\
\text { TMDinit (name) was } \\
\text { determined }\end{array}$ \\
\hline $\begin{array}{l}\text { TMDgetQmin ( ) } \\
\text { (TMDgetQ2min ( ) ) }\end{array}$ & $\begin{array}{l}\text { Returns the minimum value } \\
\text { of the energy scale } \mu \text { (in } \\
\mathrm{GeV}),\left(\mu^{2}\left(\mathrm{in}_{\mathrm{GeV}}^{2}\right)\right) \text { for } \\
\text { dataset }\end{array}$ \\
\hline $\begin{array}{l}\text { TMDgetQmax ( ) } \\
\text { (TMDgetQ2max ( ) ) }\end{array}$ & $\begin{array}{l}\text { Returns the maximum value } \\
\text { of the energy scale } \mu \text { (in } \\
\mathrm{GeV}),\left(\mu^{2}\left(\text { in } \mathrm{GeV}^{2}\right)\right) \text { for } \\
\text { dataset }\end{array}$ \\
\hline TMDgetExtrapolation_Q2 () & $\begin{array}{l}\text { Returns the method of } \\
\text { extrapolation in scale } \\
\text { outside the grid definition } \\
\text { as specified in info file }\end{array}$ \\
\hline TMDgetExtrapolation_kt () & $\begin{array}{l}\text { Returns the method of } \\
\text { extrapolation in } k_{t} \text { outside } \\
\text { the grid definition as } \\
\text { specified in info file }\end{array}$ \\
\hline TMDgetExtrapolation_x() & $\begin{array}{l}\text { Returns the method of } \\
\text { extrapolation in } x \text { outside } \\
\text { the grid definition as } \\
\text { specified in info file }\end{array}$ \\
\hline TMDnumberPDF (name) & $\begin{array}{l}\text { Returns the identifier as a } \\
\text { value of the associated } \\
\text { name of the dataset }\end{array}$ \\
\hline TMDstringPDF (index) & $\begin{array}{l}\text { Returns the name associated } \\
\text { with index of the dataset }\end{array}$ \\
\hline
\end{tabular}

\subsection{TMDlib calling sequence}

In the following simple examples are given to demonstrate how information from the TMD parton densities can be obtained in $\mathrm{C}++$ and Fortran.

\section{- in $\mathrm{C}++$}

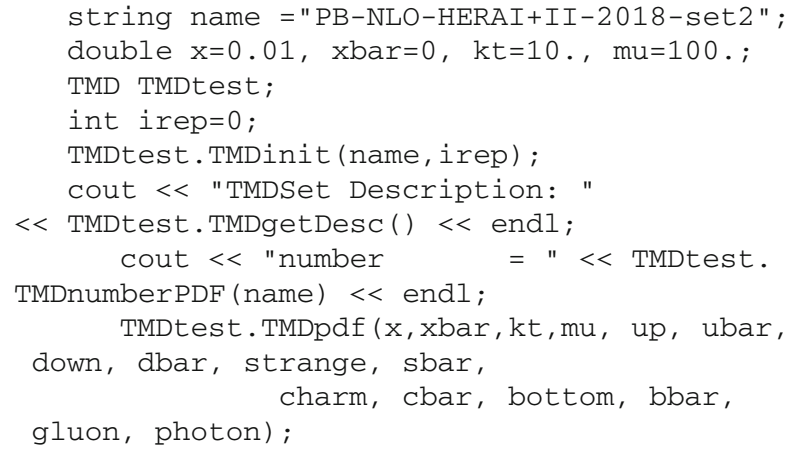

- in Fortran (using multiple replicas of the TMD)

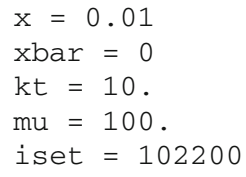

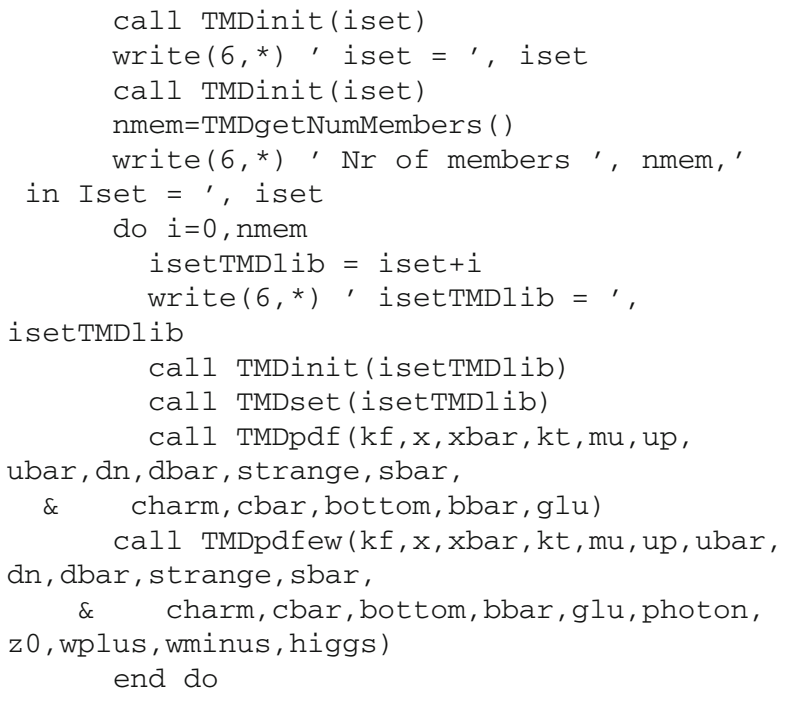

\subsection{Installation of TMD grids}

The TMD grid files are no longer automatically distributed with the code package, but have to be installed separately. A list of available TMD parameterizations is given in Table 1 .

\# get help

bin/TMDlib-getdata --help

\# install all data sets

bin/TMDlib-getdata all

\# install a single data (for example: SV19_nnlo) bin/TMDlib-getdata SV19_nnlo

\subsection{Structure of TMD grids}

In TMDlib2 the TMDgrids are stored in directories with the name of a given TMD set which is located in installation_prefix/share/tmalib/TMDset Name. Every such directory contains info file and grid file(s), for example for a TMD set called test:

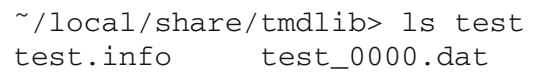

The info file contains general information on the TMDset (inspired by a similar strategy in LHAPDF), as described below, and the file(s) test_0000. dat contains the TMDgrid. If further replicas are available (for example for uncertainties), the files are numbered as test_0000.dat, test_0001.dat, ..., with the number of files given by NumMembers as described below.

The info file must contain all the information to initialize and use the TMDgrid: 


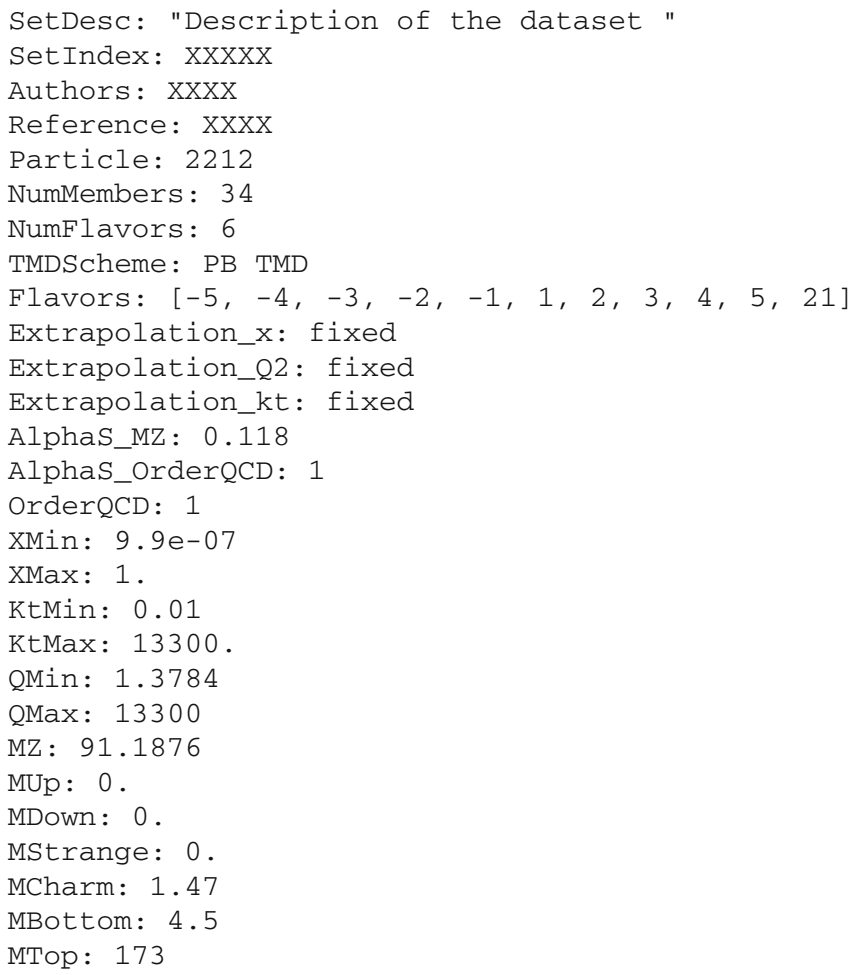

The meaning of most entries is obvious from their name, with TMDScheme different structures for the TMD grids can be selected:

\author{
PB TMD used for the PB TMD series \\ PB TMD-EW used for the PB TMD series including elec- \\ troweak particles \\ Pavia TMDs used for the PaviaTMD (or similar TMD) \\ series
}

\section{Summary}

The authors of this manual set up a collaboration to develop and maintain TMDlib and TMDplotter, respectively a $\mathrm{C}++$ library for handling different parameterizations of uPDFs/ TMDs and a corresponding online plotting tool. The aim is to update these tools with more uPDF/TMD parton sets and new features, as they become available and are developed. TMDlib2 improves on the efficiency of previous versions, allows for simpler $\mathrm{C}++$ interfaces and simplifies the inclusion of new uPDF/TMD sets.

Acknowledgements N. Abdulov was also supported by the RFBR Grant 19-32-90096. S. Baranov, A. Lipatov and M. Malyshev are grateful the DESY Directorate for the support in the framework of Cooperation Agreement between MSU and DESY on phenomenology of the LHC processes and TMD parton densities. V. Bertone is supported by the European Union's Horizon 2020 research and innovation programme under Grant agreement No 824093. C. Bissolotti is supported by the European Research Council (ERC) under the European
Union's Horizon 2020 research and innovation program (Grant Agreement No. 647981, 3DSPIN) and by the U.S. Department of Energy contract DE-AC05-06OR23177 under which Jefferson Science Associates operates the Thomas Jefferson National Accelerator Facility. F. Hautmann thanks DESY, Hamburg and CERN, Theory Group for hospitality and support. M. Hentschinski gratefully acknowledges support by Consejo Nacional de Ciencia y Tecnología Grant Number A1 S43940 (CONACYT-SEP Ciencias Básicas). A. Lelek acknowledges funding by Research Foundation-Flanders (FWO) (application number: $1272421 \mathrm{~N})$. M. Malyshev and N. Abdulov were supported by the grant of the Foundation for the Advancement of Theoretical Physics and Mathematics (Grants 20-1-3-11-1 and 18-1-5-33-1, respectively. A. Signori acknowledges support from the European Commission through the Marie Skłodowska-Curie Action SQuHadron (Grant Agreement ID: 795475). S. Taheri Monfared thanks the Humboldt Foundation for the Georg Forster research fellowship and gratefully acknowledges support from IPM. A. van Hameren acknowledges support from the Polish National Science Centre Grant No. 2019/35/ST2/03531. K. Kutak acknowledges the support by Polish National Science Centre Grant No. DEC-2017/27/B/ST2/01985 Q. Wang and H. Yang acknowledge the support by the Ministry of Science and Technology under Grant No. 2018YFA040390 and by the National Natural Science Foundation of China under Grant No. 11661141008

Data Availability Statement This manuscript has no associated data or the data will not be deposited. [Authors' comment: The source is available from https://tmdlib.hepforge.org/. The data grid files are availabel from the DESY cloud: https://syncandshare.desy.de/index.php/s/ GjjcwKQC93M979e?path=\%2FTMD\%20grid\%20files.]

Open Access This article is licensed under a Creative Commons Attribution 4.0 International License, which permits use, sharing, adaptation, distribution and reproduction in any medium or format, as long as you give appropriate credit to the original author(s) and the source, provide a link to the Creative Commons licence, and indicate if changes were made. The images or other third party material in this article are included in the article's Creative Commons licence, unless indicated otherwise in a credit line to the material. If material is not included in the article's Creative Commons licence and your intended use is not permitted by statutory regulation or exceeds the permitted use, you will need to obtain permission directly from the copyright holder. To view a copy of this licence, visit http://creativecomm ons.org/licenses/by/4.0/.

Funded by $\mathrm{SCOAP}^{3}$.

\section{References}

1. V.N. Gribov, L.N. Lipatov, Sov. J. Nucl. Phys. 15, 438 (1972)

2. V.N. Gribov, L.N. Lipatov, Yad. Fiz. 15, 781 (1972)

3. G. Altarelli, G. Parisi, Nucl. Phys. B 126, 298 (1977)

4. Y.L. Dokshitzer, Sov. Phys. JETP 46, 641 (1977)

5. Y.L. Dokshitzer, Zh Eksp, Teor. Fiz. 73, 1216 (1977)

6. R. Angeles-Martinez et al., Acta Phys. Polon. B 46, 2501 (2015). arXiv: 1507.05267

7. J.C. Collins, D.E. Soper, Nucl. Phys. B 193, 381 (1981) [Erratum: Nucl. Phys. B 213, 545 (1983)]

8. J.C. Collins, D.E. Soper, Nucl. Phys. B 194, 445 (1982)

9. J.C. Collins, D.E. Soper, G.F. Sterman, Nucl. Phys. B 223, 381 (1983)

10. J.C. Collins, D.E. Soper, G.F. Sterman, Phys. Lett. B 109, 388 (1982)

11. J.C. Collins, D.E. Soper, G.F. Sterman, Nucl. Phys. B 250, 199 (1985) 
12. J. Collins, Foundations of Perturbative QCD, Vol. 32. Cambridge Monographs on Particle Physics, Nuclear Physics and Cosmology (2011)

13. R. Meng, F.I. Olness, D.E. Soper, Phys. Rev. D 54, 1919 (1996). arXiv:hep-ph/9511311

14. P.M. Nadolsky, D.R. Stump, C.P. Yuan, Phys. Rev. D 61, 014003 (2000). arXiv:hep-ph/9906280 [Erratum: Phys. Rev. D 64, 059903 (2001)]

15. P.M. Nadolsky, D.R. Stump, C.P. Yuan, Phys. Rev. D 64, 114011 (2001). arXiv:hep-ph/0012261

16. X.-D. Ji, J.-P. Ma, F. Yuan, Phys. Rev. D 71, 034005 (2005). arXiv:hep-ph/0404183

17. X.-D. Ji, J.-P. Ma, F. Yuan, Phys. Lett. B 597, 299 (2004). arXiv:hep-ph/0405085

18. M.G. Echevarria, A. Idilbi, I. Scimemi, JHEP 07, 002 (2012). arXiv: 1111.4996

19. J.-Y. Chiu, A. Jain, D. Neill, I.Z. Rothstein, Phys. Rev. Lett. 108, 151601 (2012). arXiv: 1104.0881

20. S. Catani, M. Ciafaloni, F. Hautmann, Phys. Lett. B 242, 97 (1990)

21. S. Catani, M. Ciafaloni, F. Hautmann, Nucl. Phys. B 366, 135 (1991)

22. E.M. Levin, M.G. Ryskin, Y.M. Shabelski, A.G. Shuvaev, Sov. J. Nucl. Phys. 53, 657 (1991)

23. J.C. Collins, R.K. Ellis, Nucl. Phys. B 360, 3 (1991)

24. S. Catani, M. Ciafaloni, F. Hautmann, Phys. Lett. B 307, 147 (1993)

25. S. Catani, F. Hautmann, Nucl. Phys. B 427, 475 (1994). arXiv:hep-ph/9405388

26. F. Hautmann, Phys. Lett. B 535, 159 (2002). arXiv:hep-ph/0203140

27. E. Avsar (2012). arXiv:1203.1916

28. E. Avsar, Int. J. Mod. Phys. Conf. Ser. 04, 74 (2011). arXiv: 1108.1181

29. S. Jadach, M. Skrzypek, Acta Phys. Polon. B 40, 2071 (2009). arXiv:0905.1399

30. F. Dominguez, Unintegrated Gluon Distributions at Small-x. Ph.D. Thesis, Columbia University (2011)

31. F. Dominguez, J.-W. Qiu, B.-W. Xiao, F. Yuan, Phys. Rev. D 85, 045003 (2012). arXiv: 1109.6293

32. F. Dominguez, A. Mueller, S. Munier, B.-W. Xiao, Phys. Lett. B 705, 106 (2011). arXiv: 1108.1752

33. F. Hautmann, Acta Phys. Polon. B 40, 2139 (2009)

34. F. Hautmann, M. Hentschinski, H. Jung (2012). arXiv:1205.6358

35. F. Hautmann, H. Jung, Nucl. Phys. Proc. Suppl. 184, 64 (2008). arXiv:0712.0568

36. F. Hautmann, H. Jung, A. Lelek, V. Radescu, R. Zlebcik, JHEP 01, 070 (2018). arXiv:1708.03279

37. F. Hautmann, H. Jung, A. Lelek, V. Radescu, R. Zlebcik, Phys. Lett. B 772, 446 (2017). arXiv: 1704.01757

38. B.R. Webber, Annu. Rev. Nucl. Part. Sci. 36, 253 (1986)

39. R.K. Ellis, W.J. Stirling, B.R. Webber, Camb. Monogr. Part. Phys. Nucl. Phys. Cosmol. 8, 1 (1996)

40. F. Hautmann, H. Jung, M. Krämer, P. Mulders, E. Nocera et al., Eur. Phys. J. C 74, 3220 (2014). arXiv: 1408.3015

41. P. Connor, H. Jung, F. Hautmann, J. Scheller, PoS DIS2016, 039 (2016)

42. H. Plothow-Besch, Comput. Phys. Commun. 75, 396 (1993)

43. H. Plothow-Besch, Int. J. Mod. Phys. A 10, 2901 (1995)

44. A. Buckley, J. Ferrando, S. Lloyd, K. Nordström, B. Page, M. Rüfenacht, M. Schönherr, G. Watt, Eur. Phys. J. C 75, 132 (2015). arXiv: 1412.7420

45. A Bermudez Martinez, P. Connor, F. Hautmann, H. Jung, A. Lelek, V. Radescu, R. Zlebcik, Phys. Rev. D 99, 074008 (2019). arXiv: 1804.11152

46. ZEUS, H1 Collaboration, H. Abramowicz et al., Eur. Phys. J. C 75, 580 (2015). arXiv: 1506.06042

47. A. Bacchetta, F. Delcarro, C. Pisano, M. Radici, A. Signori, JHEP 06, 081 (2017). arXiv:1703.10157
48. L.A. Harland-Lang, A.D. Martin, P. Motylinski, R.S. Thorne, Eur. Phys. J. C 75, 204 (2015). arXiv:1412.3989

49. M.A. Ebert, B. Mistlberger, G. Vita, JHEP 09, 146 (2020). arXiv:2006.05329

50. M.-X. Luo, T.-Z. Yang, H.X. Zhu, Y.J. Zhu, Phys. Rev. Lett. 124, 092001 (2020). arXiv: 1912.05778

51. G. Watt, A.D. Martin, M.G. Ryskin, Eur. Phys. J. C 31, 73 (2003). arXiv:hep-ph/0306169

52. F. Hautmann, Phys. Lett. B 655, 26 (2007). arXiv:hep-ph/0702196

53. F. Hautmann, Phys. Lett. B 643, 171 (2006). arXiv:hep-ph/0610078

54. J.C. Collins, T.C. Rogers, A.M. Stasto, Phys. Rev. D 77, 085009 (2008). arXiv:0708.2833

55. G. Watt, A.D. Martin, M.G. Ryskin, Phys. Rev. D 70, 014012 (2004). arXiv:hep-ph/0309096

56. J. Collins, H. Jung (2005). arXiv:hep-ph/0508280

57. V. Bertone, PoS 2017, DIS201 (2018). arXiv:1708.00911

58. V. Bertone, S. Carrazza, J. Rojo, Comput. Phys. Commun. 185 1647 (2014). arXiv: 1310.1394

59. J. Pumplin, D. Stump, J. Huston, H. Lai, P.M. Nadolsky et al., JHEP 0207, 012 (2002). arXiv:hep-ph/0201195

60. W.T. Giele, S. Keller, Phys. Rev. D 58, 094023 (1998). hep$\mathrm{ph} / 9803393$

61. W.T. Giele, S.A. Keller, D.A. Kosower (2001). arXiv:hep-ph/0104052

62. ApfelWeb. https://apfel.mi.infn.it/

63. Durham PDFplotter. http://hepdata.cedar.ac.uk/pdf/pdf3.html

64. R. Brun, F. Rademakers, Nucl. Instrum. Meth. A 389, 81 (1997)

65. H. Jung (2004). arXiv:hep-ph/0411287

66. M. Hansson, H. Jung (2003). arXiv:hep-ph/0309009

67. F. Hautmann, H. Jung, Nucl. Phys. B 883, 1 (2014). arXiv: 1312.7875

68. N.A. Abdulov, H. Jung, A.V. Lipatov, G.I. Lykasov, M.A. Malyshev, Phys. Rev. D 98, 054010 (2018). arXiv:1806.06739

69. A.V. Kotikov, A.V. Lipatov, B.G. Shaikhatdenov, P. Zhang, JHEP 02, 028 (2020). arXiv:1911.01445

70. H. Jung, S.T. Monfared, T. Wening, Phys. Lett. B 817, 136299 (2021). arXiv:2102.01494

71. E. Blanco, A. van Hameren, H. Jung, A. Kusina, K. Kutak, Phys. Rev. D 100, 054023 (2019). arXiv:1905.07331

72. K.J. Golec-Biernat, M. Wusthoff, Phys. Rev. D 59, 014017 (1998). arXiv:hep-ph/9807513

73. J. Blumlein, On the $k_{T}$ dependent gluon density in hadrons and in the photon, in ' $95 Q C D$ and high-energy hadronic interactions. Proceedings, 30th Rencontres de Moriond, Moriond Particle Physics Meetings, Hadronic Session, Le Arcs, France, March 19-25, 1995, pp. 191-197 (1995). Also in preprint arXiv:hep-ph/9506446

74. K. Kutak, S. Sapeta, Phys. Rev. D 86, 094043 (2012). arXiv: 1205.5035

75. K. Kutak, Phys. Rev. D 91, 034021 (2015). arXiv:1409.3822

76. P. Kotko, K. Kutak, S. Sapeta, A.M. Stasto, M. Strikman, Eur. Phys. J. C 77, 353 (2017). arXiv:1702.03063

77. M.G. Echevarria, T. Kasemets, P.J. Mulders, C. Pisano, JHEP 07, 158 (2015). arXiv: 1502.05354

78. M. Bury, A. van Hameren, H. Jung, K. Kutak, S. Sapeta, M. Serino, Eur. Phys. J. C 78, 137 (2018). arXiv: 1712.05932

79. A. Signori, A. Bacchetta, M. Radici, G. Schnell, JHEP 1311, 194 (2013). arXiv:1309.3507

80. A. Bacchetta, V. Bertone, C. Bissolotti, G. Bozzi, F. Delcarro, F. Piacenza, M. Radici, JHEP 07, 117 (2020). arXiv:1912.07550

81. A. Bacchetta, F. Delcarro, C. Pisano, M. Radici (2020). arXiv:2004.14278

82. I. Scimemi, A. Vladimirov (2019). arXiv:1912.06532

83. A. Vladimirov, JHEP 10, 090 (2019). arXiv:1907.10356

84. M. Bury, A. Prokudin, A. Vladimirov, Phys. Rev. Lett. 126, 112002 (2021). arXiv:2012.05135 